\title{
Clinical and laboratory features that discriminate dengue from other febrile illnesses: a diagnostic accuracy study in Rio de Janeiro, Brazil
}

Regina P Daumas ${ }^{1 *}$, Sonia RL Passos², Raquel VC Oliveira², Rita MR Nogueira ${ }^{3}$, Ingebourg Georg ${ }^{4}$, Keyla BF Marzochi ${ }^{5}$ and Patrícia Brasil ${ }^{5}$

\begin{abstract}
Background: Dengue is an acute febrile illness caused by an arbovirus that is endemic in more than 100 countries Early diagnosis and adequate management are critical to reduce mortality. This study aims to identify clinical and hematological features that could be useful to discriminate dengue from other febrile illnesses (OFI) up to the third day of disease.

Methods: We conducted a sectional diagnostic study with patients aged 12 years or older who reported fever lasting up to three days, without any evident focus of infection, attending an outpatient clinic in the city of Rio de Janeiro, Brazil, between the years 2005 and 2008. Logistic regression analysis was used to identify symptoms, physical signs, and hematological features valid for dengue diagnosis. Receiver-operating characteristic (ROC) curve analyses were used to define the best cut-off and to compare the accuracy of generated models with the World Health Organization (WHO) criteria for probable dengue.

Results: Based on serological tests and virus genome detection by polymerase chain reaction (PCR), 69 patients were classified as dengue and 73 as non-dengue. Among clinical features, conjunctival redness and history of rash were independent predictors of dengue infection. A model including clinical and laboratory features (conjunctival redness and leukocyte counts) achieved a sensitivity of $81 \%$ and specificity of $71 \%$ and showed greater accuracy than the WHO criteria for probable dengue.

Conclusions: We constructed a predictive model for early dengue diagnosis that was moderately accurate and performed better than the current WHO criteria for suspected dengue. Validation of this model in larger samples and in other sites should be attempted before it can be applied in endemic areas.
\end{abstract}

Keywords: Dengue/diagnosis, Signs and symptoms, Sensitivity and specificity, Fever/diagnosis

\section{Background}

Dengue is an acute febrile illness caused by an arbovirus (arthropod-borne virus) transmitted mainly by Aedes aegypti mosquitoes. Four dengue virus serotypes (DENV-1 to 4 ) are recognized and a primary infection by one serotype does not provide immunity against the others [1]. Nowadays, dengue is a global public health problem. It is endemic in more than 100 countries

\footnotetext{
* Correspondence: regina.daumas@gmail.com

${ }^{1}$ Germano Sinval Faria Teaching Primary Care Center/National School of Public Health, Oswaldo Cruz Foundation - Fiocruz, Rio de Janeiro 21041-210, Brazil

Full list of author information is available at the end of the article
}

where 50 to 100 million infections are estimated to occur each year [2].

In the region of Americas, Brazil alone accounted for more than $60 \%$ of reported cases from 2000 to 2007, when epidemics occurred in different regions of the country related to the co-circulation of DENV-1, DENV-2, and DENV-3 [3]. The state of Rio de Janeiro was the site of introduction and later dissemination of these serotypes after epidemics in the years 1986, 1990, and 2002 [4]. In $2007 / 2008$, DENV-2 was reintroduced in this state and caused an epidemic with more severe clinical presentations and more fatalities than previous ones, primarily among children and adolescents [3]. Recently, DENV-4

\section{Ciomed Central}


reemerged in the country, being identified in the state of Rio de Janeiro in 2011 [5].

Clinical presentation of dengue fever varies along a wide spectrum of signs and symptoms. Typically, it presents as a self-limiting disease characterized by fever associated with symptoms such as headache, nausea, vomiting, arthralgia, myalgia and/or rash. However, during the course of the disease, some patients develop severe manifestations related to increased vascular permeability and plasma leakage that can lead to death. Signs of spontaneous bleeding may be present and are more frequent in severe forms [6]. There is no specific therapy, but timely initiated supportive treatment can reduce the lethality of severe cases to less than 1\% [7].

Early identification of dengue infection could help clinicians to institute adequate case management and to identify patients who should be closely monitored for signs of plasma leakage. This information might promote early supportive therapies [8], prevent the use of potentially harmful drugs [9] and encourage assessment of prognosis and the use of treatment guidelines [10].

Despite the recent development of rapid laboratory tests [11-13], their availability is still limited and most diagnoses in endemic areas are based on clinical and epidemiological criteria. Until 2006, most studies on clinical features to discriminate dengue from other febrile illnesses have evaluated hospitalized patients in Southeast Asia [14]. Despite some evidence that clinical signs and symptoms vary during the course of the disease, few of those studies reported disease duration at the time of clinical evaluation. Therefore, comparison of results and identification of useful features for early diagnosis was difficult [14].

In recent years, the focus on early clinical predictors $[15,16]$ and the use of regression methods to evaluate multiple predictors has increased [15-17]. Some studies using these methods were also done in the Americas [18-21]. In Brazil, although some studies have identified signs and symptoms associated with confirmed dengue cases [22,23], the evaluation of multiple clinical criteria with the aim of constructing a diagnostic algorithm has not yet been attempted.

This study aims to: 1) identify clinical and laboratory features useful for discriminating dengue from other febrile illnesses (OFI) in the first three days of disease; and 2) evaluate the accuracy of combinations of these features.

\section{Methods}

\section{Settings and participants}

A sectional diagnostic study was conducted with data that were systematically collected at the outpatient clinic for Acute Febrile Illnesses (AFI) of a public research institute from January 2005 to July 2008. Data collected once a week by the same staff in the Emergency Department (ED) of a public general hospital from November 2007 to January 2008 were also included. Patients older than 12 years of age who presented with a history of fever lasting up to three days without an evident or suspected focus on clinical examination (such as tonsillitis or pyelonephritis) were eligible to be included in the study. Those with severely compromised health status (i. e. with altered consciousness, signs of shock or severely dehydrated) in need of emergency care were excluded. Enrollment was sequential at the outpatient clinic, four afternoons a week. At the emergency department, once a week the research team evaluated the first eight eligible patients who attended the ED. Both health units are located in the city of Rio de Janeiro and the research institute does not provide pediatric care. Although dengue fever is considered endemic in Rio de Janeiro, from November 2007 to April 2008 a DEN-2 epidemic was also detected in the city.

\section{Collection of patient data}

Attending staff included two infectious disease specialists and two supervised medical students. All were trained to perform and record standardized clinical evaluations and fill a form containing 28 symptoms and 25 signs potentially useful in the differential diagnosis of febrile illnesses, according to literature review and specialists consensus. Interobserver agreement on signs and symptoms was evaluated in a subsample of 140 patients and was almost perfect (kappa >0.80) for most collected clinical data [24]. The questionnaire is available online for consultation and details on its development have been described elsewhere [24].

On initial presentation, patients were evaluated through comprehensive clinical history taking and physical examination. Clinical data were recorded on the standardized form and blood samples were collected for a complete blood count and dengue diagnostic tests. A second sample was requested for all patients 7-10 days after the initial visit for paired serology. Additional laboratory tests were done according to individual clinical judgment.

The study was approved by the IPEC-FIOCRUZ Ethics Committee and all the patients and/or their guardians agreed to participate by providing a written informed consent.

\section{Variable definitions}

Clinical variables investigated were standardized according to clear definitions described in the research proto$\mathrm{col}$ and included the following: hepatomegaly - liver size of $12 \mathrm{~cm}$ or more at percussion; lymph node enlargement - presence of one or more lymph nodes measuring $1 \mathrm{~cm}$ or more at palpation; pallor - any degree of skin 
or mucosa paleness; dehydration - dry mucosa at inspection; and splenomegaly - a palpable spleen at inspiration with the patient lying on his right side. Taste disorder was considered present when patients answered affirmatively to the question "Does food or water taste differently?"

\section{Laboratory methods}

Acute and convalescent serum samples were tested for anti-dengue immunoglobulin M (IgM). Blood samples collected up to the fifth febrile day were also screened for viral RNA using reverse-transcriptase polymerase chain reaction (RT-PCR) and/or tested for NS1 antigen. Light microscopy of thick stained blood smears for intracellular malarial parasites was performed in acute blood samples from patients who had been in malaria endemic regions.

Tests for IgM detection were done using an IgM antibody-capture enzyme-linked immunosorbent assay (MAC-ELISA) (PanBio, Brisbane, Australia) according to the manufacturer's instructions. Dengue NS1 antigen was detected with the Platelia ${ }^{\mathrm{TM}}$ Dengue NS1Ag-ELISA (Bio-Rad Laboratories Marnes-la-Coquette, France). Both tests were performed at the Laboratory of Immunodiagnostics at IPEC-FIOCRUZ. RNA detection was performed in the Laboratory of Flavivirus, Oswaldo Cruz Institute (FIOCRUZ) using RT-PCR according to Lanciotti et al. [25].

\section{Case definitions}

Dengue diagnosis was based on the combination of different tests, considering their accuracy in different disease phases [1,26-28]. Stating as day 0 the day of fever onset, we classified patients as:

Dengue (D): A patient who tests positive for either IgM antibodies, viral RNA (RT-PCR) or NS1 antigen;

Non-Dengue (ND): A patient who tests negative for anti-dengue IgM antibodies in a sample collected between day 7 and day 21 OR a patient with a unique sample collected between day 0 and day 2 that is negative for both NS1 antigen and anti-dengue IgM;

Indeterminate: A patient who is negative for IgM antibodies and NS1 antigen in an acute sample collected after day 2 for whom a convalescent sample is not available.

\section{Statistical analysis}

We carried out an exploratory analysis of clinical and laboratory features comparing D and ND groups. We used Pearson Chi-square or Fisher's exact test for categorical and Mann-Whitney test for continuous variables, considering a level of significance of 0.05 . Variables that were significant in the exploratory analysis were selected for inclusion in a multiple logistic regression model. The two estimated models included: a) clinical data only, and b) clinical and laboratory data.

Logistic regression parameters were estimated with the Firth procedure that uses the penalized likelihood function [29]. This method enables estimation of parameters in small samples with strongly predictive covariates, where the phenomenon of separation makes at least one parameter estimate diverge to \pm infinity. A manual stepwise backward selection approach was used, considering as the criterion for maintaining variables in the model a p-value of less than 0.05 on the penalized log-likelihood ratio test.

For the regression model including laboratory data, a receiver-operating characteristic (ROC) curve was plotted. The best cut-off value for probabilities was determined by the Youden index that maximizes the sum of sensitivity and specificity. Subsequently, these values were substituted in the model's equation and the combinations of variable values that classified a patient as a dengue case were determined.

We also evaluated the predictive accuracy of the WHO criteria for probable dengue, which states that at least two of the following manifestations must be present in an acute febrile patient: headache, retroorbital pain, myalgia, arthralgia, exanthema, leukopenia, and hemorrhagic manifestations [30]. For each patient, we computed the number of these manifestations (from zero to seven) that were present. We defined leukopenia as a leukocyte count lower than $4,000 / \mathrm{mm}^{3}$.

With the aim of ascertaining the best predictive model, ROC curve analysis was also done for WHO criteria and the difference between the areas under the curves (AUC) of the curves' pair (estimated model $x$ WHO criteria) was tested by Delong's method [31]. Confidence intervals (CI) for the AUC, sensitivities and specificities were estimated using pROC package functions which compute the $95 \%$ CI with 2000 stratified bootstrap replicates (with replacement) [32]. Data collected in forms were entered using Epidata 3.1 software [33]. Exploratory analyses were done with Statistical Package for Social Sciences - SPSS v. 17.0 (SPSS Inc., Chicago, Illinois). Logistic regression with Firth correction was implemented with logistf package [34] and ROC curve analyses with $p R O C$ package [32] from the R 2.11.1 software [35].

\section{Results}

From a total of 182 evaluated patients, 40 (22.0\%) were excluded from analyses as their diagnoses were indeterminate. From the remaining 142 patients, 69 were classified as dengue (D) and 73 as non-dengue (ND). Median age and distribution by health units were not different between D and ND patients. Male sex was predominant (65.2\%) among dengue cases, but was not among nondengue patients $(47.9 \%)$. The majority of dengue cases 
(71\%) were enrolled in the epidemic period (Table 1). DENV-3 was the unique identified serotype in the first years of the study, whereas DENV-2 was firstly isolated in January 2008 and became the predominant serotype since then (data not shown).

The most frequent clinical features in dengue patients were exhaustion (98.6\%), myalgia (92.8\%), and headache (87.0\%). However, these features were also very prevalent among ND patients and were useless for discriminating between D and ND patients. From clinical history, only taste disorder (OR: 2.15; IC95\%:1.06-4.36) and the report of rash (OR: 3.33; IC95\%: 1.56-7.07) were associated with dengue diagnosis. Bitter taste and diminished taste were the most frequently reported taste disorders. On physical examination, rash, lymph node enlargement, and conjunctival redness were significantly more frequent in D patients than in ND patients (Table 2). Considering laboratory results, platelet and leukocyte counts were significantly smaller in the D than in the ND group (Table 3).

The variables that were significantly associated with dengue diagnosis in the univariate analysis were selected for inclusion in multiple regression models. These models were based on data from 97 patients $(52 \mathrm{D}$ and 45 $\mathrm{ND)}$ as the others had incomplete data for one or more of the selected covariates. The results of multiple regression analyses are presented in Table 4. In the model including clinical data only (Model 1), conjunctival redness and history of rash were the variables independently associated with the diagnosis. At the best cut-off value, it showed an $84.6 \%$ sensitivity and $66.7 \%$ specificity. According to the model equation, this accuracy is achieved when we classify patients with conjunctival redness or exanthema as D and those with neither of these features as ND.

Table 1 Characteristics of the sample according to diagnosis of dengue or non-dengue

\begin{tabular}{|c|c|c|c|c|c|}
\hline & \multirow{2}{*}{\multicolumn{2}{|c|}{$\begin{array}{l}\text { Dengue } \\
(\mathbf{N}=69) \\
\end{array}$}} & \multirow{2}{*}{\multicolumn{2}{|c|}{$\begin{array}{c}\text { Non-dengue } \\
(\mathbf{N}=\mathbf{7 3})\end{array}$}} & \multirow[t]{2}{*}{$p$ value $^{a}$} \\
\hline & & & & & \\
\hline \multicolumn{6}{|l|}{ Sex, n (\%) } \\
\hline Male & 45 & $(65.2)$ & 35 & $(47.9)$ & 0.028 \\
\hline Female & 24 & $(34.8)$ & 38 & $(52.1)$ & \\
\hline Age in years, median (IR) & 31.0 & $(23-41)$ & 33.2 & $(24-39)$ & NS \\
\hline \multicolumn{6}{|l|}{ Period, n (\%) } \\
\hline Epidemic & 49 & $(71.0)$ & 37 & $(50.7)$ & 0.010 \\
\hline Endemic & 20 & $(29.0)$ & 36 & $(49.3)$ & \\
\hline \multicolumn{6}{|l|}{ Health unit, n (\%) } \\
\hline IPEC & 62 & $(89.9)$ & 62 & $(84.9)$ & NS \\
\hline HMLJ & 7 & $(10.1)$ & 11 & (15.1) & \\
\hline
\end{tabular}

NOTE. $-{ }^{a}$ Chi-square test for categorical variables and Mann-Whitney test for continuous variables; IR = Interquartile range.
Table 2 Clinical features associated to dengue diagnosis in patients evaluated up to $\mathbf{3}$ days after fever onset

\begin{tabular}{|c|c|c|c|c|c|}
\hline \multirow[t]{2}{*}{ Clinical data } & \multicolumn{2}{|c|}{ Dengue } & \multicolumn{2}{|c|}{ Non-dengue } & \multirow[t]{2}{*}{ OR $(95 \% \mathrm{Cl})$} \\
\hline & $\mathrm{N}$ & $\%$ & $\mathrm{~N}$ & $\%$ & \\
\hline \multicolumn{6}{|l|}{ History: } \\
\hline Exhaustion & 68 & 98.6 & 69 & 95.8 & \\
\hline Myalgia & 64 & 92.8 & 67 & 93.1 & \\
\hline Headache & 60 & 87.0 & 64 & 87.7 & \\
\hline Lumbar pain & 59 & 85.5 & 59 & 81.9 & \\
\hline Anorexia & 55 & 79.7 & 55 & 75.3 & \\
\hline Retro-orbital pain & 49 & 71.0 & 44 & 60.3 & \\
\hline Taste disorder & 47 & 70.1 & 36 & 52.2 & $2.15(1.06-4.36)$ \\
\hline Nausea & 45 & 65.2 & 40 & 54.8 & \\
\hline Arthralgia & 44 & 63.8 & 43 & 58.9 & \\
\hline Photophobia & 38 & 55.9 & 35 & 47.9 & \\
\hline Chills & 33 & 47.8 & 42 & 57.5 & \\
\hline Dizziness & 33 & 47.8 & 34 & 46.6 & \\
\hline History of rash & 30 & 44.1 & 14 & 19.2 & $3.33(1.56-7.07)$ \\
\hline Cough & 25 & 36.2 & 26 & 35.6 & \\
\hline Diarrhea & 25 & 36.2 & 18 & 24.7 & \\
\hline Vomiting & 22 & 31.9 & 17 & 23.6 & \\
\hline Itching & 21 & 31.3 & 13 & 18.6 & \\
\hline Abdominal pain & 21 & 30.4 & 31 & 42.5 & \\
\hline Coryza & 20 & 29.0 & 21 & 28.8 & \\
\hline Sore throat & 16 & 23.2 & 27 & 37.0 & \\
\hline Nasal congestion & 14 & 20.9 & 17 & 24.6 & \\
\hline History of bleeding & 12 & 17.4 & 6 & 8.2 & \\
\hline Earache & 11 & 16.2 & 5 & 6.9 & \\
\hline Hoarseness & 11 & 16.2 & 9 & 12.5 & \\
\hline Dyspnea & 10 & 14.5 & 7 & 9.7 & \\
\hline \multicolumn{6}{|l|}{ Physical exam: } \\
\hline Rash & 48 & 71.6 & 31 & 45.6 & $3.02(1.48-6.16)$ \\
\hline Conjunctival redness & 38 & 55.9 & 20 & 29.0 & $3.10(1.53-6.29)$ \\
\hline Lymph node enlargement & 33 & 50.8 & 14 & 20.6 & $3.98(1.86-8.53)$ \\
\hline Dehydration & 24 & 35.3 & 16 & 23.2 & \\
\hline Pharyngeal erythema & 15 & 22.4 & 21 & 31.3 & \\
\hline Pallor & 11 & 16.4 & 15 & 21.7 & \\
\hline Petechiae & 9 & 13.4 & 4 & 5.9 & \\
\hline Hepatomegaly & 7 & 10.4 & 5 & 7.4 & \\
\hline Splenomegaly & 5 & 7.5 & 5 & 7.2 & \\
\hline
\end{tabular}

Crude odds ratios (OR) and respective $95 \%$ confidence intervals (Cl) are shown for those with statistical significance.

In the logistic regression with clinical and laboratory data, only conjunctival redness and leukocyte count showed to be independently associated with the diagnosis and were selected to remain in the final model (Model 2). This model showed an AUC ROC of 0.82 
Table 3 Clinical signs and hematologic parameters according to definite diagnosis (dengue or non-dengue)

\begin{tabular}{|c|c|c|c|}
\hline & \multicolumn{2}{|c|}{ Median (IR) } & \multirow[t]{2}{*}{$p$ value $^{a}$} \\
\hline & Dengue & Non-dengue & \\
\hline \multirow[t]{2}{*}{ Axillary temperature $\left({ }^{\circ} \mathrm{C}\right)$} & 37.1 & 37 & NS \\
\hline & $(36.5-38.2)$ & $(36.5-37.8)$ & \\
\hline \multirow[t]{2}{*}{ Heart rate } & 84 & 80 & NS \\
\hline & $(78.0-90.0)$ & $(72.0-91.0)$ & \\
\hline \multirow[t]{2}{*}{ Respiratory rate } & 19 & 19 & NS \\
\hline & $(16.0-20.0)$ & $(16.0-20.0)$ & \\
\hline \multirow[t]{2}{*}{ MAP sitting (mmHg) } & 92.5 & 88.3 & NS \\
\hline & $(83.3-100.0)$ & $(77.5-96.7)$ & \\
\hline \multirow[t]{2}{*}{ MAP recumbent (mmHg) } & 93.3 & 93.3 & NS \\
\hline & $(83.3-102.5)$ & $(83.3-97.9)$ & \\
\hline \multirow[t]{2}{*}{ Hematocrit (\%) } & 42 & 42.3 & NS \\
\hline & $(39.3-45.0)$ & $(40.0-45.1)$ & \\
\hline \multirow[t]{2}{*}{ Platelets $\left(1000 / \mathrm{mm}^{3}\right)$} & 175 & 209 & 0.002 \\
\hline & $(121.5-218.0)$ & $(171.0-237.5)$ & \\
\hline \multirow[t]{2}{*}{ Hemoglobin (g/dl) } & 14.4 & 14.2 & NS \\
\hline & $(13.2-15.3)$ & $(13.6-15.3)$ & \\
\hline \multirow[t]{2}{*}{ Leukocytes $\left(/ \mathrm{mm}^{3}\right)$} & 3600 & 6205 & $<0.001$ \\
\hline & $(2745-5015)$ & (4248-8718) & \\
\hline
\end{tabular}

NOTE. $-\mathrm{IR}=$ Interquartile range. ${ }^{\text {a }}$ Non-parametric Mann-Whitney test. $\mathrm{MAP}=$ Mean arterial pressure

with $80.8 \%$ sensitivity and $71.1 \%$ specificity at the best cut-off value. Adjusted odds ratios for each model variable are shown on Table 4. WHO criterion for probable dengue-the presence of two out of seven featuresshowed $98.0 \%$ sensitivity but only $4.4 \%$ specificity. The ROC curve for the WHO criteria showed an AUC of 0.71 , which was significantly smaller than that of Model $2(\mathrm{p}=0.04)$ (Figure 1).

Replacing the best cut-off value in the Model 2 equation, we found two combinations of variable values that would indicate a dengue diagnosis in this setting:

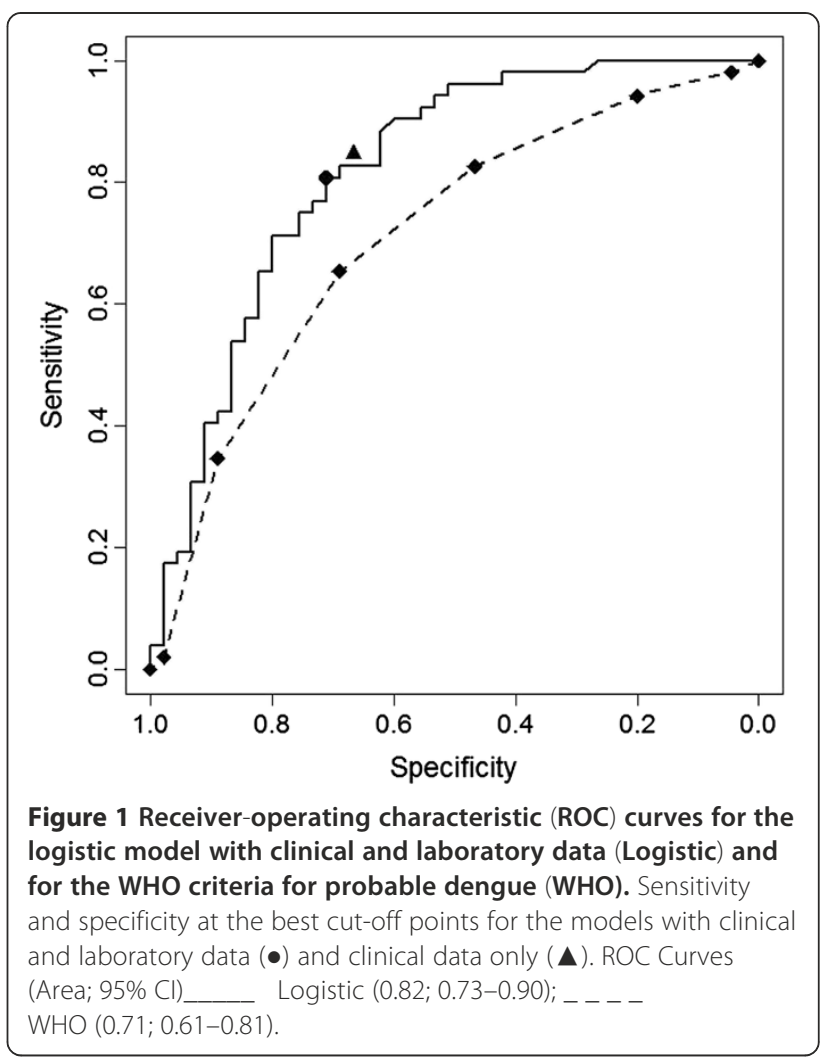

1) conjunctival redness in a patient with a leukocyte count below $7,500 / \mathrm{mm}^{3}$; or 2 ) leukocyte counts below $3,760 / \mathrm{mm}^{3}$ independently of other features.

\section{Discussion}

Dengue is one of the most underreported tropical diseases [1]. On the other hand, during epidemics, overreporting can occur at some health sites. The lack of laboratory resources and the nonspecific clinical presentation of non-severe cases greatly contribute to this situation [36]. Diagnostic algorithms based on clinical data may enhance disease diagnosis and surveillance in endemic areas.

Table 4 Adjusted odds ratios and accuracy parameters for dengue diagnosis of multiple logistic regression models according to the range of included data (clinical only or clinical and laboratory)

\begin{tabular}{|c|c|c|c|c|c|c|}
\hline & \multicolumn{2}{|c|}{ Adjusted diagnostic odds ratio } & \multicolumn{2}{|c|}{ Sensitivity } & \multicolumn{2}{|c|}{ Specificity } \\
\hline & OR & $95 \% \mathrm{Cl}$ & $\%$ & $95 \% \mathrm{Cl}$ & $\%$ & $95 \% \mathrm{Cl}$ \\
\hline \multicolumn{7}{|c|}{ Model 1 - Clinical data only } \\
\hline History of Rash & 3.84 & $1.54-10.24$ & 84.6 & $46.8-93.0$ & 66.7 & $38.6-78.8$ \\
\hline Conjunctival redness & 4.05 & $1.69-10.31$ & & & & \\
\hline \multicolumn{7}{|c|}{ Model 2 - Clinical and laboratory data } \\
\hline Conjunctival redness & 4.1 & $1.61-11.22$ & 80.8 & $59.6-94.6$ & 71.1 & $53.3-84.4$ \\
\hline Leukocytes (In) ${ }^{a}$ & 0.13 & $0.04-0.34$ & & & & \\
\hline
\end{tabular}

NOTE. - OR: odds ratio; $95 \% \mathrm{Cl}$ : $95 \%$ confidence interval. ${ }^{a}$ Effect for each unit of natural logarithm of leukocyte count. 
In this study, we identified some clinical and hematological features associated to confirmed dengue cases. We also constructed simple predictive models for dengue diagnosis based on clinical and hematological data for patients presenting early in the course of disease. These models showed moderate accuracy [37], and performed better than the diagnostic features proposed by WHO for probable dengue [30] in the study population.

Among clinical features, rash, taste disorder, conjunctival redness, and lymph node enlargement were all associated with dengue diagnosis. Rash is among the classic signs of dengue fever and its description includes different cutaneous manifestations, such as a diffuse erythema coincident with fever or a macular exanthema that is more frequent after the third day of disease $[1,6]$. Rash has also been reported to be more common in primary than in secondary infections [38], an issue that we could not explore in our study.

The extraordinarily high prevalence of rash detected on the physical exam of dengue patients $(72 \%)$ in our study can be related to major efforts to ascertain any kind of cutaneous manifestations in a research setting. These may have included a mild flushing that would otherwise be unnoticed. It should be noted that a history of rash was more accurate for dengue diagnosis than its detection on physical exam. The same finding was also reported by Chadwick et al. [17] who found that history of rash was the only independent clinical predictor that remained in a regression model which included clinical and laboratory data. In this context, the possibility that self-examination could be more accurate than medical examination at ascertaining some mild cutaneous manifestation should be considered.

Comparing our results with other reports, we found that taste alteration, conjunctival redness, and lymphadenopathy, although described as common manifestations of dengue fever [1], are not consistently investigated as predictive signs and symptoms in studies on dengue diagnosis [14]. Nevertheless, taste alteration was reported as a dengue predictor by the few researchers who investigated this symptom [15,39], and conjunctival redness was reported by Low et al. [15] as one of the most accurate signs $(\mathrm{p}<0.0005$; OR $=4.49)$ for dengue diagnosis among adult outpatients with a fever lasting less than 72 hours. The frequency of lymph node enlargement in dengue patients has varied from 3\% [14] to about $20 \%[17,23]$ in studies that reported this sign. In these studies, it was not a useful sign to discriminate $\mathrm{D}$ from ND patients, despite being more frequent among the first ones in all of them.

The importance of ocular findings warrants better investigation as recent studies have described a variety of ocular manifestations in dengue [40-42], suggesting that eye involvement may be more common than usually appreciated. Gregory et al. (2010) also highlighted the importance of ocular manifestations in dengue as they found retro-orbital pain as an important clinical feature to discriminate dengue from OFI in all age groups [20].

Among hematological data, leukocyte count was the most discriminant feature. Although common in other viral illness, leukopenia has been consistently reported as an independent predictor of dengue diagnosis among febrile patients $[14,16,19]$, particularly in adults $[19,20,43-46]$, and seems to occur earlier than thrombocytopenia $[43,44]$. Indeed, it was the most important isolated predictor in our study.

Other authors have already proposed models and scales to predict dengue infection in febrile adults living in the Americas. Comparison between predictive rules and WHO criteria through ROC curve analysis was also done by some of them. In Colombia, Díaz et al. (2006) developed a scale which performed better as an early predictor in adults than the one produced with WHO criteria (AUC ROC of $81.0 \%$ versus $70.0 \%$ ). It was composed by the presence of rash, positive tourniquet test, absence of nasal discharge, arthralgias, absence of diarrhea ( 1 point for each finding), leukocyte count $<4,000 / \mathrm{mm}^{3}$ (3 points) and platelet count $<180.000 / \mathrm{mm}^{3}$ ( 2 points) [19]. Similar accuracy was obtained in Puerto Rico with a predictive model for adults with suspected dengue (AUC ROC: 79.7\%). Retro-orbital pain, rash, absence of sore throat, and leukopenia were the independent predictors in this age group [20].

As strengths of this study, we may cite the collection of clinical data by a trained team, before the performance of laboratory tests, and the use of a comprehensive standardized protocol for history taking and examination. The recording of clinical features prior to any test result minimized the risk of observation bias, and the inclusion of clinical features other than the usual dengue signs and symptoms allowed us to identify some potentially useful new predictors.

This study also has some limitations. The first one is related to the small sample size used in multiple regression analysis. This may be attributed in part to the characteristics of our clinic, which attends mainly referred patients, to a long period without epidemics when we had slow subject enrollment and to our option to analyze only data from patients in the first three days of disease. The small sample size precluded any subgroup analyses.

We also had considerable losses because of indeterminate diagnosis (40/182), as only 66 out of 182 (36.3\%) eligible patients collected two blood samples. Although the use of NS1 test helped to diagnose dengue in patients with samples collected from day 0 to day 2, those with only one negative sample collected between 
day 3 and day 7 were classified as indeterminate, as a negative result in both IgM and NS1 could be a falsenegative by this time. As a consequence, we had relatively few confirmed ND patients. This kind of problem, however, affects most studies with outpatients, in such a way that losses greater than $30 \%$ for indeterminate diagnoses are common in this setting $[20,47]$.

We cannot exclude the possibility of some misclassification by our reference standard in dengue diagnosis. Although sensitivities above $90 \%$ for NS1 on days 0 to 2 $[26,48]$ and sensitivities above $93 \%$ for IgM in samples collected after the seventh day of disease have been reported in patients with a primary infection $[1,28,49]$, both tests have lower sensitivities in secondary infections $[28,50]$. As dengue is endemic in Rio de Janeiro, it is possible that some dengue patients have been classified as non-dengue. The consequence of this eventual misclassification would be a decrease in the measured odds ratios with underestimation of the accuracy of analyzed predictors.

The interpretation and generalization of the results of studies such as this one must consider the fact that diagnostic accuracy of clinical manifestations also depends on their frequency in the non-dengue group. This means that it varies according to the incidence of other febrile illnesses (OFI) in the same period and place. For instance, low platelet counts may be useless to discriminate dengue from OFI in a place where malaria is endemic, as low platelet counts are also frequent in the later [17]. In our study, laboratory tests for OFI were required as indicated by clinical suspicion and we are unable to describe the prevalence of other diagnoses, except for malaria, which was rare (2\%) in the study population.

Although our model outperformed the diagnostic accuracy of the WHO criteria, its predictive value is still poor, with some $20 \%$ false negative among dengue patients and 30\% false positive among those with OFI. The low accuracy of WHO case definition, mainly because of its low specificity has been described by Martinez et al. (2005), who also explored the accuracy of different number of WHO criteria [51]. The difficulty to identify early clinical predictors of dengue infection in adults has been described by Ramos et al. (2009) in a large study in Puerto Rico. Although they identified eye pain, diarrhea and absence of upper respiratory symptoms as independently associated to confirmed dengue cases, they also highlighted the low predictive value of these features, alone or in combination, for early infection in adults [18]. In spite of this relatively low accuracy, the use of management protocols based on clinical diagnostic scales proved useful to reduce hospitalizations due to dengue in Colombia [52].

Nonetheless, the results of this study are potentially helpful for surveillance in adults, as they suggest that the proposed criteria, derived from simple clinical and hematological data, can be more accurate than the criteria for reporting suspected dengue cases currently in use. The use of this alternative algorithm could enhance the ascertainment of dengue cases by clinical and epidemiological criteria, and enable a more accurate estimation of the disease burden. Meanwhile, it should be stressed that in endemic areas, while early accurate laboratory tests are not widely available, dengue fever should be considered in every patient presenting with an acute undifferentiated febrile illness. Monitoring all these patients for the development of signs of severity, however, may impose a great burden on the health services. Once validated, algorithms that enable early identification of dengue cases could influence clinical outcomes as they would allow closely monitoring of selected patients. This procedure may warrant timely identification of alarm signs and the adoption of simple and widely available therapeutic support measures that are effective in preventing fatalities [7].

\section{Conclusions}

We constructed predictive models for early dengue diagnosis that were moderately accurate and performed better than the diagnostic features proposed by WHO for dengue surveillance.

Considering only clinical features, the presence of conjunctival redness or a history of skin rash, in contrast with the absence of these features, showed $84.6 \%$ sensitivity and $66.7 \%$ specificity for dengue diagnosis. Conjunctival redness in a patient with a leukocyte count below $7,500 / \mathrm{mm}^{3}$ or leukocyte counts below $3,760 / \mathrm{mm}^{3}$ independently of other features showed an $80.8 \%$ sensitivity and $71.1 \%$ specificity.

These results can be useful for the development of predictive clinical tools as they may call attention to signs that are frequently ignored on dengue research. Validation of the proposed predictive model in larger datasets is advisable before using it for clinical or surveillance purposes. Studies with large sample sizes are also needed to identify and confirm predictors of dengue infection in different epidemiological contexts.

\section{Abbreviations \\ OFI: Other febrile illnesses; ROC: Receiver-operating characteristic; PCR: Polymerase chain reaction; DEN-V: Dengue virus; OFI: Other febrile illnesses; AFI: Acute febrile illnesses; WHO: World Health Organization; IgM: Immunoglobulin M; RNA: Ribonucleic acid; RT-PCR: Reverse-transcriptase polymerase chain reaction; MAC-ELISA: IgM antibody-capture enzyme-linked immunosorbent assay; D: Dengue case; ND: Non-dengue case; ED: Emergency department; AUC: Area under the curve.}

Competing interests

The authors declare that they have no competing interests.

\section{Authors' contributions}

RPD participated in the design of the study, performed the statistical analysis and drafted the manuscript. RVCO participated in statistical analysis. RMRN 
carried out molecular biology assays. IG carried out the immunoassays. SRLP, PB and KBFM conceived the study, participated in its design and coordination and helped to draft the manuscript. All authors revised the manuscript, contributed to its content, read and approved its final version.

\section{Acknowledgements}

We are grateful to: Liliane A. Reis and Marlene da Conceição Rezende for administrative support; Patrícia Rosana de Souza, Diego V. Carneiro, Bruno B. G. Carvalho and Clarice S. Bressan for contribution in data collection; João Claudio Arnaldo Alves e Flávia Lattario Ribeiro for help with data entry; emergency medical staff, laboratory staff, and the epidemiology service team coordinated by Mayumi Wakimoto for all support provided at Lourenço Jorge Municipal Hospital.

\section{Author details}

${ }^{1}$ Germano Sinval Faria Teaching Primary Care Center/National School of Public Health, Oswaldo Cruz Foundation - Fiocruz, Rio de Janeiro 21041-210, Brazil. 'Laboratory of Clinical Epidemiology/Evandro Chagas Clinical Research Institute, Oswaldo Cruz Foundation - Fiocruz, Rio de Janeiro 21040-361, Brazil. ${ }^{3}$ Flavivirus Laboratory, Department of Virology, Instituto Oswaldo Cruz/ FIOCRUZ, 21040-190 Rio de Janeiro, Brazil. ${ }^{4}$ Immunology Service/Evandro Chagas Clinical Research Institute, Oswaldo Cruz Foundation - Fiocruz, Rio de Janeiro 21040-361, Brazil. ${ }^{5}$ Laboratory of Acute Febrile Illnesses/Evandro Chagas Clinical Research Institute, Oswaldo Cruz Foundation - Fiocruz, Rio de Janeiro, Brazil.

Received: 23 July 2012 Accepted: 4 February 2013

Published: 8 February 2013

\section{References}

1. Gubler DJ: Dengue and dengue hemorrhagic fever. Clin Microbiol Rev 1998, 11:480-496.

2. Guzman A, Isturiz RE: Update on the global spread of dengue. Int J Antimicrob Agents 2010, Suppl 1:S40-S42.

3. San Martin JL, Brathwaite O, Zambrano B, Solorzano JO, Bouckenooghe A, Dayan GH, Guzman MG: The epidemiology of dengue in the americas over the last three decades: a worrisome reality. AmJTrop Med Hyg 2010, 82:128-135.

4. Nogueira RM, de Araujo JM, Schatzmayr HG: Dengue viruses in Brazil, 1986-2006. Rev Panam Salud Publica 2007, 22:358-363.

5. Nogueira RM, Eppinghaus AL: Dengue virus type 4 arrives in the state of Rio de Janeiro: a challenge for epidemiological surveillance and control. Memorias do Instituto Oswaldo Cruz 2011, 106:255-256.

6. Rigau-Perez JG, Clark GG, Gubler DJ, Reiter P, Sanders EJ, Vorndam AV: Dengue and dengue haemorrhagic fever. Lancet 1998, 352:971-977.

7. $\mathrm{WHO}($ World Health Organization: Dengue: guidelines for diagnosis, treatment, prevention and control - New edition. Newth edition. Geneva: WHO; 2009.

8. Harris E, Perez L, Phares CR, Perez Mde L, Idiaquez W, Rocha J, Cuadra R, Hernandez E, Campos LA, Gonzales A, et al: Fluid intake and decreased risk for hospitalization for dengue fever, Nicaragua. Emerg Infect Dis 2003, 9:1003-1006.

9. Diaz-Quijano FA, Villar-Centeno LA, Martinez-Vega RA: Effectiveness of early dipyrone administration on severity of dengue virus infection in a prospective cohort. Enferm Infecc Microbiol Clin 2005, 23:593-597.

10. Diaz-Quijano FA, Villar-Centeno LA, Martinez-Vega RA: Predictors of spontaneous bleeding in patients with acute febrile syndrome from a dengue endemic area. J Clin Virol 2010, 49:11-15.

11. Nga TT, Thai KT, Phuong HL, Giao PT, Hung Le Q, Binh TQ, Mai VT, Van Nam N, De Vries PJ: Evaluation of two rapid immunochromatographic assays for diagnosis of dengue among Vietnamese febrile patients. Clin Vaccine Immunol 2007, 14:799-801.

12. Shu PY, Yang CF, Kao JF, Su CL, Chang SF, Lin CC, Yang WC, Shih H, Yang SY, Wu PF, et al: Application of the dengue virus NS1 antigen rapid test for on-site detection of imported dengue cases at airports. Clin Vaccine Immunol 2009, 16:589-591.

13. Zainah S, Wahab AH, Mariam M, Fauziah MK, Khairul AH, Roslina I, Sairulakhma A, Kadimon SS, Jais MS, Chua KB: Performance of a commercial rapid dengue NS1 antigen immunochromatography test with reference to dengue NS1 antigen-capture ELISA. J Virol Methods 2009, 155:157-160
14. Potts JA, Rothman AL: Clinical and laboratory features that distinguish dengue from other febrile illnesses in endemic populations. Trop Med Int Health 2008, 13:1328-1340

15. Low JG, Ooi EE, Tolfvenstam T, Leo YS, Hibberd ML, Ng LC, Lai YL, Yap GS, Li CS, Vasudevan SG, Ong A: Early Dengue infection and outcome study (EDEN) - study design and preliminary findings. Ann Acad Med Singapore 2006, 35:783-789.

16. Tanner L, Schreiber M, Low JG, Ong A, Tolfvenstam T, Lai YL, Ng LC, Leo YS, Thi Puong L, Vasudevan SG, et al: Decision tree algorithms predict the diagnosis and outcome of dengue fever in the early phase of illness. PLoS Negl Trop Dis 2008, 2:e196.

17. Chadwick D, Arch B, Wilder-Smith A, Paton N: Distinguishing dengue fever from other infections on the basis of simple clinical and laboratory features: application of logistic regression analysis. J Clin Virol 2006, 35:147-153.

18. Ramos MM, Tomashek KM, Arguello DF, Luxemburger C, Quinones L, Lang J, Munoz-Jordan JL: Early clinical features of dengue infection in Puerto Rico. Trans R Soc Trop Med Hyg 2009, 103:878-884.

19. Diaz FA, Martinez RA, Villar LA: [Clinical criteria to diagnose dengue in its early stages]. Biomedica: revista del Instituto Nacional de Salud 2006, 26:22-30.

20. Gregory CJ, Santiago LM, Arguello DF, Hunsperger E, Tomashek KM: Clinical and laboratory features that differentiate dengue from other febrile illnesses in an endemic area-Puerto Rico, 2007-2008. AmJTrop Med Hyg 2010, 82:922-929.

21. Biswas HH, Ortega O, Gordon A, Standish K, Balmaseda A, Kuan G, Harris E: Early clinical features of dengue virus infection in nicaraguan children: a longitudinal analysis. PLoS Negl Trop Dis 2012, 6:e1562.

22. Nunes-Araujo FR, Ferreira MS, Nishioka SD: Dengue fever in Brazilian adults and children: assessment of clinical findings and their validity for diagnosis. Ann Trop Med Parasitol 2003, 97:415-419.

23. Passos SR, Bedoya SJ, Hokerberg YH, Maia SC, Georg I, Nogueira RM, Souza RV, Marzochi KB: Clinical and laboratory signs as dengue markers during an outbreak in Rio de Janeiro. Infection 2008, 36:570-574.

24. Daumas RP, Brasil P, Bressan CS, Oliveira RV, Carvalho BB, Carneiro DV, Passos SR: Interobserver agreement on signs and symptoms of patients with acute febrile illness. Infection 2011, 39:135-140.

25. Lanciotti RS, Calisher CH, Gubler DJ, Chang GJ, Vorndam AV: Rapid detection and typing of dengue viruses from clinical samples by using reverse transcriptase-polymerase chain reaction. J Clin Microbiol 1992, 30:545-551.

26. Chuansumrit A, Chaiyaratana W, Pongthanapisith V, Tangnararatchakit K, Lertwongrath S, Yoksan S: The use of dengue nonstructural protein 1 antigen for the early diagnosis during the febrile stage in patients with dengue infection. Pediatr Infect Dis J 2008, 27:43-48

27. Guzman MG, Jaenisch T, Gaczkowski R, Ty Hang VT, Sekaran SD, Kroeger A Vazquez S, Ruiz D, Martinez E, Mercado JC, et al: Multi-country evaluation of the sensitivity and specificity of two commercially-available NS1 ELISA assays for dengue diagnosis. PLoS Negl Trop Dis 2010, 4(8):e811.

28. Sa-Ngasang A, Anantapreecha S, A-Nuegoonpipat A, Chanama S, Wibulwattanakij S, Pattanakul K, Sawanpanyalert P, Kurane I: Specific IgM and IgG responses in primary and secondary dengue virus infections determined by enzyme-linked immunosorbent assay. Epidemiol Infect 2006, 134:820-825.

29. Heinze $G$, Schemper M: A solution to the problem of separation in logistic regression. Stat Med 2002, 21:2409-2419.

30. WHO (World Health Organization: Dengue haemorrhagic fever: diagnosis, treatment, prevention and control. 2nd edition. Geneva: WHO; 1997.

31. DeLong ER, DeLong DM, Clarke-Pearson DL: Comparing the areas under two or more correlated receiver operating characteristic curves: a nonparametric approach. Biometrics 1988, 44:837-845.

32. Robin X, Turck N, Hainard A, Tiberti N, Lisacek F, Sanchez J-C, Müller M: pROC: Display and analyze ROC curves v.1.3.2. http://cran.r-project.org/web/ packages/pROC.

33. Lauritsen JM, Bruus M: (2003-2004) EpiData (version 3). A comprehensive tool for validated entry and documentation of data. Odense, Denmark: The EpiData Association; http://www.epidata.dk/.

34. Ploner M, Dunkler D, Southworth H, Heinze G: logistf: Firth's bias reduced logistic regression. R package version 1.10. http://cran.r-project.org/web/ packages/logistf. 
35. R Development Core Team: R: A Language and Environment for Statistical Computing. Vienna, Austria: R Foundation for Statistical Computing; 2010

36. Phuong HL, De Vries PJ, Nga TT, Giao PT, Hung Le Q, Binh TQ, Nam NV, Nagelkerke N, Kager PA: Dengue as a cause of acute undifferentiated fever in Vietnam. BMC Infect Dis 2006, 6:123.

37. Swets JA: Measuring the accuracy of diagnostic systems. Science 1988, 240:1285-1293.

38. Pancharoen C, Mekmullica J, Thisyakorn U: Primary dengue infection: what are the clinical distinctions from secondary infection? Southeast Asian J Trop Med Public Health 2001, 32:476-480.

39. McBride WJ, Mullner H, LaBrooy JT, Wronski I: The 1993 dengue 2 epidemic in Charters Towers, North Queensland: clinical features and public health impact. Epidemiol Infect 1998, 121:151-156

40. Seet RC, Quek AM, Lim EC: Symptoms and risk factors of ocular complications following dengue infection. J Clin Virol 2007, 38:101-105.

41. Kapoor HK, Bhai S, John M, Xavier J: Ocular manifestations of dengue fever in an East Indian epidemic. Can J Ophthalmol 2006, 41:741-746.

42. Teoh SCB, Chan DPL, Nah GKM, Rajagopalan R, Laude A, Ang BSP, Timothy BT, Cheeb KLC, Lima TH, Goha KY: A re-look at ocular complications in dengue fever and dengue hemorrhagic fever. Dengue Bull 2006 , 30:184-193.

43. Binh PT, Matheus S, Huong VT, Deparis X, Marechal V: Early clinical and biological features of severe clinical manifestations of dengue in Vietnamese adults. J Clin Virol 2009, 45:276-280.

44. Deparis X, Murgue B, Roche C, Cassar O, Chungue E: Changing clinical and biological manifestations of dengue during the dengue-2 epidemic in French Polynesia in 1996/97-description and analysis in a prospective study. Trop Med Int Health 1998, 3:859-865.

45. Hammond SN, Balmaseda A, Perez L, Tellez Y, Saborio SI, Mercado JC, Videa $E$, Rodriguez $Y$, Perez MA, Cuadra $R$, et al: Differences in dengue severity in infants, children, and adults in a 3-year hospital-based study in Nicaragua. AmJTrop Med Hyg 2005, 73:1063-1070.

46. Gregory CJ, Lorenzi OD, Colon L, Garcia AS, Santiago LM, Rivera RC, Bermudez LJ, Baez FO, Aponte DV, Tomashek KM, et al: Utility of the tourniquet test and the white blood cell count to differentiate dengue among acute febrile illnesses in the emergency room. PLoS Negl Trop Dis 2011, 5:e1400

47. Martinez-Vega RA, Diaz-Quijano FA, Villar-Centeno LA: Low concordance between early clinical suspicion of dengue and its serological confirmation. Rev Med Chil 2006, 134:1153-1160

48. Lima Mda R, Nogueira RM, Schatzmayr HG, dos Santos FB: Comparison of three commercially available dengue NS1 antigen capture assays for acute diagnosis of dengue in Brazil. PLoS Negl Trop Dis 2010, 4:e738.

49. Blacksell SD, Doust JA, Newton PN, Peacock SJ, Day NP, Dondorp AM: A systematic review and meta-analysis of the diagnostic accuracy of rapid immunochromatographic assays for the detection of dengue virus IgM antibodies during acute infection. Trans R Soc Trop Med Hyg 2006, 100:775-784.

50. Kumarasamy V, Chua SK, Hassan Z, Wahab AH, Chem YK, Mohamad M, Chua KB: Evaluating the sensitivity of a commercial dengue NS1 antigencapture ELISA for early diagnosis of acute dengue virus infection. Singapore Med J 2007, 48:669-673.

51. Martinez RA, Diaz FA, Villar LA: Evaluation of the World Health Organization clinical definition of dengue. Biomedica: revista del Instituto Nacional de Salud 2005, 25:412-416.

52. Diaz-Quijano FA, Villar-Centeno LA, Martinez-Vega RA: Reducing hospitalization with the use of a dengue management algorithm in Colombia. Rev Panam Salud Publica 2011, 30:248-254.

doi:10.1186/1471-2334-13-77

Cite this article as: Daumas et al: Clinical and laboratory features that discriminate dengue from other febrile illnesses: a diagnostic accuracy study in Rio de Janeiro, Brazil. BMC Infectious Diseases 2013 13:77.

\section{Submit your next manuscript to BioMed Central and take full advantage of:}

- Convenient online submission

- Thorough peer review

- No space constraints or color figure charges

- Immediate publication on acceptance

- Inclusion in PubMed, CAS, Scopus and Google Scholar

- Research which is freely available for redistribution 